\title{
PAPER
}

\section{Migraine with unilateral motor symptoms: a case-control study}

\author{
William B Young, Kaanchan S Gangal, Raoul J Aponte, Ronald S Kaiser
}

See Editorial Commentary, p 553

J Neurol Neurosurg Psychiatry 2007;78:600-604. doi: 10.1136/jnnp.2006.100214

See end of article for authors' affiliations

.......................

Correspondence to: Dr W B Young, Jefferson Headache Center, 111 South Eleventh Street, Suite \#8130, Philadelphia, PA 19107, USA; william.b. young@jefferson.edu

Received 20 June 2006 Revised 29 September 2006 Accepted 6 October 2006 Published Online First 20 October 2006 .....................
Objective: To characterise the clinical features of non-familial migraine with unilateral motor symptoms (MUMS) and compare these features with those of migraine without weakness.

Methods: 24 patients with MUMS and 48 matched controls were identified from a tertiary care headache centre. Using a structured interview, the migraine symptoms of both groups were characterised. Results of previously administered Beck Depression Inventories (BDI), Minnesota Multiphasic Personality Inventories and psychiatric diagnoses were collected, when available, and compared between groups.

Results: 9 patients had episodic migraine and 15 had chronic migraine. Patients with MUMS always had weakness involving the arm subjectively, and both arm and leg objectively. A give-way character was always present. Only 17\% of patients with MUMS reported facial weakness; $58 \%$ reported persistent interictal weakness; $92 \%$ reported sensory symptoms. A rostrocaudal march of sensory and motor symptoms was frequently reported. Weakness was ipsilateral to unilateral headache in two thirds of the patients. Compared with controls, patients with MUMS had had similar pain intensities, but were more likely to report other migrainous symptoms, including allodynia. $38 \%$ of patients with MUMS were told they had had a stroke, and $17 \%$ believed they had had a stroke despite normal brain imaging. Patients with MUMS reported fewer affective disorders and more adjustment disorders than controls, and had similar BDI scores.

Conclusions: A syndrome of severe migraine with accompanying give-way weakness is common in tertiary care headache centres. It is accompanied by other neurological symptoms.
M igraine has a variety of subtypes, some of which are associated with hemiplegia. Aura is highly variable and is thought to be generated from many areas of the cortex or brain stem. A recent epidemiological study estimated the prevalence of both familial and sporadic hemiplegic migraine in Denmark to be $0.01 \% .{ }^{1}$ By contrast, Couch et $a l^{2}$ reported the rate of hemiplegic symptoms to be $10 \%$ in a large tertiary care headache practice. In all, 11 of 78 patients admitted to the Thomas Jefferson University Hospital (Philadelphia, Pennsylvania, USA) for the treatment of headache reported hemiplegic symptoms, and six had hemiparesis on admission. ${ }^{3}$

Of 205 subjects in Fisher's studies of patients with late-life migraine accompaniments, 45 had motor weakness. ${ }^{45}$ All cases were accompanied by visual symptoms, paraesthesias and speech disturbances. Of 22 cases of migraine and cluster headache with limb pain accompanying the headache, six also had recurrent weakness. ${ }^{6}$ Sensorimotor disturbances often accompany chronic pain syndromes. Complex regional pain syndrome is often associated with allodynia, weakness and dystonia. ${ }^{7}$ These symptoms, along with non-dermatomal sensory loss, have been labelled "psychogenic pseudoneurological dysfunction" by some authors. ${ }^{7}$ Motor impairments correlate with allodynia in complex regional pain syndrome type $\mathrm{I}^{8}$

Our study was designed to confirm and further characterise previous observations on motor weakness accompanying migraine headache. We postulated that unilateral motor symptoms are common in patients with migraine in a tertiary care centre, and that their presence may be due to the activation of sensorimotor programme related to the pain and presence of allodynia.

\section{METHODS}

Outpatients who had a diagnosis of either episodic migraine or chronic migraine and self-reported unilateral weakness accompanying their headaches and were seen at the Jefferson Headache Center (Philadelphia, Pennnsylvania, USA) from June to September 2004 were enrolled. For each case, we entered two controls with the same headache diagnosis and a similar appointment time with the same doctor and nurse practitioner. The International Headache Society (IHS) ${ }^{9}$ criteria were used to classify patients with episodic migraine, and the 1996 Silberstein-Lipton criteria were used for patients with $\geqslant 15$ headache days per month, as the 2006 revisions to the IHS criteria for chronic migraine were not published at the time of the study. Patients were excluded if they had an abnormal magnetic resonance imaging scan, had participated in the previous MUMS study, had a family history of hemiplegic migraine or showed inconsistencies on examination. Headache was rated on the 11 -point oral severity scale $(0-10)$. Symptoms of allodynia were assessed using five questions (box). A migraine disability assessment scale was administered to each subject, along with four other disability-related questions. Brush allodynia was tested by brushing subjects with a twicefolded $4 \times 4$ gauze pad 10 times at $2 \mathrm{~Hz}$ on the bilateral forehead, cheek and neck. Give-way weakness was defined as a sudden loss of resistance during muscle strength testing of at least two sites on one side of the body.

Beck Depression Inventory (BDI-II) scores, Minnesota Multiphasic Personality Inventory (MMPI) scores, and psychiatric diagnoses (DSM-IV) assigned by a psychologist or a psychiatrist (performed routinely at the patient's initial evaluation) were retrospectively collated. From the MMPI, we calculated the Gough F-K, to identify patients who may attempt to present themselves positively or negatively, and

Abbreviations: BDI, Beck Depression Inventory; FHM, familial hemiplegic migraine; IHS, International Headache Society; MMPI, Minnesota Multiphasic Personality Inventory; MUMS, migraine with unilateral motor symptoms; SHM, sporadic hemiplegic migraine 


\section{Box 1 Allodynia questions}

- Do any of the following bother you when you have headache

- Combing or brushing your hair

- Touching your scalp or face

- Wearing any object on your head/neck (hat, jewellery, glasses, necklaces, etc)

- Wearing any object on your arms/wrist (watch, jewellery, long sleeves, etc)

- Exposure of your face/head to wind

the conversion $\mathrm{V}$, which can indicate denial as part of their coping strategy.

$\chi^{2}$ analysis and Fisher's exact test were used to compare categorical data. Unpaired Student's t test was used for normally distributed continuous variables, and the rank sum or Mann-Whitney U test for non-normally distributed data.

The protocol was approved by the Institutional Review Board of Thomas Jefferson University.

\section{RESULTS}

We screened 28 patients who had unilateral weakness with headache; one had familial hemiplegic migraine, two had inconsistent examinations within one session and one failed to complete the interview. These patients were excluded, and thus we had 24 patients and 48 controls. Nine had a diagnosis of episodic migraine and 15 had chronic migraine. Table 1 presents the demographics of the patients and controls.

Table 2 presents the average age of onset of frequent headache (defined as $>15$ days/month), duration of both episodic migraine and chronic migraine, and the frequency and intensity of headache.

Patients with MUMS had headache both with and without weakness. Patients with MUMS with episodic migraine reported that MUMS and their usual migraine had similar intensities. Patients with chronic migraine reported that exacerbation severity for MUMS and non-MUMS headache were similar (table 3).

Of the 24 patients with MUMS, 21 (88\%) reported unilateral headaches. The weakness was always ipsilateral to the headache in $13(62 \%)$ patients, and always contralateral in 7 (33\%). The relationship between the side of weakness and headache was variable in $1(5 \%)$ patient.

\section{Signs and symptoms of weakness}

In all, $4(17 \%)$ patients with MUMS reported weakness of the face, $24(100 \%)$ reported weakness of the arm or hand, and 2 $(8 \%)$ reported leg weakness. Hemiparesis was strictly one sided

Table 1 Demographics of cases and controls

\begin{tabular}{llll}
\hline & Cases & Controls & $\mathbf{p}$ \\
\hline Age (years), mean (SD) & $43.4(12.7)$ & $39.5(10.5)$ & $\mathrm{NS}$ \\
Sex (F/M) & $22 / 2$ & $39 / 9$ & $\mathrm{NS}$ \\
Race (AA/C/H) & $3 / 21 / 0$ & $1 / 46 / 1$ & $\mathrm{NS}$ \\
Age of onset of migraine & $18.0(10.3)$ & $18.3(10.3)$ & $\mathrm{NS}$ \\
(years), mean (SD) & & & \\
Typical visual aura (Y/N) & $15 / 9$ & $7 / 41$ & $<0.001$ \\
$\begin{array}{l}\text { Age of onset of MUMS } \\
\text { (years), mean (SD) }\end{array}$ & $36.5(11.5)$ & - & - \\
\hline
\end{tabular}

$\mathrm{F}$, female; $\mathrm{M}$, male; MUMS, migraine with unilateral motor symptoms. in $16(67 \%)$ patients with MUMS, and switched sides in 8 (33\%). In addition, 19 (78\%) patients reported a "march" of weakness was rostrocaudal in $18(95 \%)$ and caudorostral in 1 $(5 \%) ; 17(71 \%)$ subjects believed treating the headache improved the weakness. In total, $14(58 \%)$ patients had persistent weakness without the headache or headache exacerbation. Weakness persisted at least half the time in 2 of $9(22 \%)$ patients with episodic migraine and in 7 of $15(47 \%)$ patients with chronic migraine. Figure 1 shows the duration of weakness or exacerbation of baseline weakness.

On examination, $12(50 \%)$ patients were found to have weakness with a give-way character at the time of examination. Only 9 of the $12(75 \%)$ patients were weak and were aware of their weakness at the time of examination. In all, 2 (17\%) patients had weakness in the face; all patients had weakness in both arm and leg. Subjects reported a median of 30 days/year with MUMS (occurrence or exacerbation of baseline weakness; range 2-265 days/year). Patients with episodic migraine reported a median of 24 days/year and those with chronic migraine 36 days/year (non-significant, Mann-Whitney U test).

\section{Other aura-like symptoms}

Of 24 patients with MUMS, 15 (63\%) met the IHS criteria for typical visual aura. Sensory symptoms were reported by 22 (92\%) patients with MUMS, with $15(64 \%)$ reporting a march of sensory symptoms. The most common descriptors were tingling and loss of feeling. In all, 22 (92\%) patients reported visual symptoms with the MUMS headache; 13 (59\%) reported phosphenes; 19 (86\%) reported blurred vision; and 5 (23\%) reported diplopia. Visual symptoms were reported as monocular by 11 of $22(50 \%)$ patients, as hemianopic by $4(18 \%)$ and as bilateral by $11(50 \%)$. Non-specific language disturbance was reported by $92 \%$ of patients, and confusion by $83 \%$. The order of MUMS symptoms was highly variable among 20 patients with MUMS who indicated their first symptom: 10 (50\%) ranked pain first, 7 (35\%) ranked visual symptoms first, 2 (20\%) ranked confusion first, l (1\%) ranked language first, and none ranked sensory or motor symptoms first.

\section{Beliefs about symptoms}

In all, $9(38 \%)$ patients reported being told by a doctor that their symptoms were due to stroke; 3 (13\%) were told they had hemiplegic migraine and 1 (4\%) was told they had psychiatric disorder. Four (17\%) patients believed they had a stroke despite normal brain imaging, whereas 11 (46\%) believed their symptoms were migraine related.

\section{Comparison of patients with MUMS with controls}

Age, sex, onset of both episodic migraine and chronic migraine, and duration of migraine and of daily illness were similar for both groups (table 2). Patients with MUMS had more symptoms of migraine and cranial autonomic activation than controls (table 4).

Neck pain was equally common in both groups. Patients with MUMS had more allodynia symptoms than did controls. Patients with MUMS were more likely to have brush allodynia in V1 (50\% v 17\%; $\mathrm{p}=0.005)$, V2 (58\% v 15\%; $\mathrm{p}<0.001)$ and C2 $(33 \%$ v 6\%; $p=0.005)$. Patients with MUMS had similar, migraine disability assessment scale scores of "highly disabled" compared with controls. About 33\% of patients with MUMS had lost their jobs because of their headaches, compared with $8.3 \%$ of controls $(p=0.011)$, and $29 \%$ of patients with MUMS reported being "housebound" by their headache, compared with $8.3 \%$ of controls $(p=0.027)$. In addition, $21 \%$ of the patients with MUMS reported not applying for jobs because of their headaches, compared with $17 \%$ of controls $(p=N S)$, and 
Table 2 Comparison of features of cases and controls

\begin{tabular}{|c|c|c|c|}
\hline & Cases & Controls & $\mathrm{p}$ Value \\
\hline \multicolumn{4}{|c|}{ Features of episodic migraine (cases 9 , control 18) } \\
\hline Days/month & $5.8(3.4)$ & $6.4(3.4)$ & NS \\
\hline No of episodes $3 /$ month & $15.2(10.3)$ & $17.3(10.2)$ & NS \\
\hline Average intensity $(0-10)$ & $7.4(1.8)$ & $5.8(2.0)$ & 0.05 \\
\hline Average duration (h) & $7.1(15.4)$ & $16.2(28.9)$ & NS \\
\hline \multicolumn{4}{|c|}{ Features of transformed migraine (cases 15, control 30) } \\
\hline Age of onset of frequent $\mathrm{HA}$, (year & $31.6(9.6)$ & $27.4(12.8)$ & NS \\
\hline No of years with frequent $\mathrm{HA}$ & $14.0(9.1)$ & $12.3(12.7)$ & NS \\
\hline No of days with HA in 3 months & $64.2(20.0)$ & $76.9(17.6)$ & NS \\
\hline Intensity of baseline HA & $4.6(4.8)$ & $4.8(1.7)$ & NS \\
\hline Continuous HA (Y/N) & $14 / 1$ & $21 / 9$ & NS \\
\hline $\begin{array}{l}\text { No of days with exacerbation in } \\
3 \text { months }\end{array}$ & $26.1(20.3)$ & $24.1(22.1)$ & NS \\
\hline $\begin{array}{l}\text { Average intensity of } \mathrm{HA} \\
\text { exacerbation }\end{array}$ & $8.7(1.4)$ & $8.4(2.0)$ & NS \\
\hline
\end{tabular}

HA, headache; MUMS, migraine with unilateral motor symptoms. Data are presented as mean (SD), unless otherwise specified. The mean age of onset of MUMS was 36.5 years (range 14-59).

8.3\% of patients with MUMS reported limiting their working hours to part time, compared with $12.5 \%$ of controls $(p=N S)$.

Patients with MUMS recalled receiving more testing than controls: the median number of remembered brain images (computed tomography or magnetic resonance imaging) was five, compared with two for controls $(\mathrm{p}=0.001$, rank sum test). Patients with MUMS were more likely than controls to recall having treatments withheld due to concerns about safety $(37.5 \% \vee 8.3 \%, \mathrm{p}=0.004$, rank sum test).

\section{Psychological evaluation in cases and controls}

Data on psychiatric evaluations were available for 18 (75\%) patients with MUMS and 45 (94\%) controls. BDI-II data were available for $16(66.7 \%)$ patients and $40(83.3 \%)$ controls. No statistically significant differences in age, sex, presence of aura, or diagnosis of episodic migraine or chronic migraine were present among patients and controls who received a psychiatric evaluation, took a BDI-II or had an available MMPI, and those who did not.

Most cases and controls had at least one psychiatric diagnosis, four cases and nine controls had two, none had more than two, one case and three controls had none. Most had a diagnosis of depression (major depression, dysthymia, bipolar with depressed mood, or adjustment disorder with depressed mood), anxiety (generalised anxiety disorder and panic disorder), both, or an adjustment disorder other than with depressed mood (fig 2). Depression was more common in controls than cases, but this was not statistically significant.

There were no significant differences in either total BDI score or in the answer to question 9 (suicide question) between groups (table 5 ).

All patients with MUMS and controls answered either 0 or 1 to the suicide question; there were no answers of 2, 3 or 4 (more suicidal) to this question. MMPI scores were similar between cases and controls across all scales (table 5). Gough scores were similar between patients with MUMS and controls (table 5). The Gough F-K is designed to identify patients who attempt to present themselves positively or negatively with scores that are negative and enter double figures, suggesting a deliberate attempt on the patient's part to present as particularly virtuous, whereas positive scores that enter double figures suggest the over-reporting of distress or pathology for reasons that include malingering or crying out for help because of emotional pain. Conversion Vs were more common among patients with MUMS than among controls, but this did not reach statistical significance when corrected for multiple comparisons. The conversion V configuration is based on the first three clinical
Table 3 Comparison of different headaches in patients with MUMS

\begin{tabular}{llll}
\hline & $\begin{array}{l}\text { Headache with } \\
\text { weakness }\end{array}$ & $\begin{array}{l}\text { Headache } \\
\text { without weakness }\end{array}$ & p Value \\
\hline $\begin{array}{l}\text { Severity of EM } \\
\begin{array}{l}\text { Duration with treatment } \\
\text { (h) }\end{array}\end{array}$ & $2.7(1.1)$ & $7.4(1.8)$ & NS \\
CM exacerbation severity & $8.1(1.5)$ & $2(7.1)$ & NS \\
\hline
\end{tabular}

$C M$, chronic migraine; EM, episodic migraine.

*Data are presented as median (mean), as they do not follow a normal distribution.

scales of the MMPI-2: scale 1 , which reflects physical complaints; scale 2, which measures depression; and scale 3, which reflects a combination of traits including denial, the inhibition of emotions, conflict avoidance and possibly conversion disorder.

\section{DISCUSSION}

Unilateral motor symptoms were common in patients with migraine at a tertiary headache centre, and symptoms were usually ipsilateral to the headache, associated with sensory complaints and spread in a rostrocaudal direction. These data, along with our previous findings that 11 of 76 inpatients with headache had a history of unilateral weakness, indicate that MUMS is an important phenomenon that requires explanation. ${ }^{3}$ Despite normal brain imaging, patients are often told, and may believe, that they have had a stroke.

The symptoms of MUMS are fairly characteristic. Motor symptoms typically begin with the onset of pain or worsen as the pain intensifies. Motor symptoms are usually accompanied by sensory symptoms. A march of motor and sensory symptoms is common. Visual symptoms can be positive or negative. Other common neurological symptoms include non-specific language disturbances and dizziness. These symptoms often meet the IHS definition of aura. Furthermore, most patients with MUMS have typical IHS-defined visual aura. Except for the distinction between true and give-way weakness, many patients with MUMS fulfill the International classification of headache disorders, 2nd edition, criteria for hemiplegic migraine. As patients will not report the difference between true weakness and give-way weakness, and as distinguishing give-way and true weakness on examination may be difficult, ${ }^{4}$ some reports of sporadic hemiplegic migraine it is likely that include patients with MUMS.

The choice of the control group is crucial to understand these results. As we controlled for the type of migraine (episodic migraine $v$ chronic migraine), our comparisons of symptoms, disability, resource use, and to some extent psychiatric

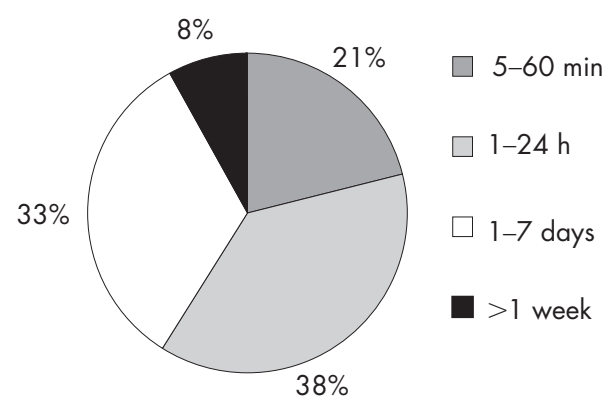

Figure 1 Pie chart showing variable duration of weakness (or exacerbation of weakness) reported by patients with migraine with unilateral motor symptoms (MUMS). 
Table 4 Symptom frequencies

\begin{tabular}{llll}
\hline Symptom & Cases & Controls & p Value* \\
\hline Pulsation & $24 / 0$ & $22 / 26$ & $<0.001$ \\
Exacerbated by exercise & $22 / 2$ & $23 / 25$ & $<0.004$ \\
Nausea & $21 / 3$ & $18 / 30$ & $<0.001$ \\
Vomiting & $13 / 11$ & $12 / 36$ & 0.326 \\
Photophobia & $24 / 0$ & $25 / 23$ & $<0.001$ \\
Phonophobia & $18 / 6$ & $21 / 27$ & 0.236 \\
Osmophobia & $15 / 9$ & $20 / 28$ & 1.000 \\
Ptosis & $16 / 8$ & $8 / 48$ & 0.001 \\
Unilateral nasal congestion & $9 / 15$ & $5 / 43$ & 0.179 \\
Bilateral nasal congestion & $7 / 17$ & $9 / 39$ & 1.000 \\
Eye redness & $11 / 13$ & $5 / 43$ & 0.029 \\
Unilateral tearing & $12 / 12$ & $4 / 44$ & $<0.002$ \\
Bilateral tearing & $9 / 15$ & $6 / 42$ & 0.479 \\
Neck pain before headache & $16 / 8$ & $28 / 20$ & 1.000 \\
Neck pain during headache & $18 / 6$ & $35 / 13$ & 1.000 \\
Neck pain after headache & $12 / 12$ & $18 / 30$ & 1.000 \\
Neck pain independent of headache & $11 / 13$ & $21 / 27$ & 1.000 \\
\hline *With Bonferroni correction for multiple comparisons. & \\
\hline
\end{tabular}

comorbidity, became more meaningful. Controlling for time and type of visit (routine follow-up, infusion and after hospitalisation) helps in selecting a control group with a similar burden of illness. Controlling for provider helps avoid recruitment biases, as different providers may accumulate different types of patients. The choice of control group makes comparisons of resource use and psychological factors more meaningful. The similarities between reported headache intensities and duration of illness suggest that this strategy is at least partly successful.

Even when compared with other disabled people with migraine, patients with MUMS can be considered to be "super migraineurs". With similar intensities of pain and duration of illness, patients with MUMS report more associated symptoms, more allodynia, and more cluster-like eye and nasal symptoms. They often have more extreme forms of disability and receive more diagnostic testing.

What is the source of the weakness? The symptoms may be due to an aura of which the mechanism is cortical spreading neuronal excitation and depression. ${ }^{10}{ }^{11}$ Alternatively, the symptoms may represent a sensorimotor phenomenon or motor behavioural syndrome, possibly related to the presence of allodynia, which has spread beyond the territory of the head pain. Finally, the weakness may represent malingering or conversion disorder.

If MUMS weakness is due to an aura, it should be considered a late-onset form of sporadic hemiplegic migraine. In our study, the ratio of MUMS to familial hemiplegic migraine was 25:1. The strong tendency of the motor symptoms to appear or worsen with severe pain and improve with treatment, and the presence of give-way weakness, are findings against aura, but the march of symptoms is suggestive.

Thomsen reviewed the symptoms of subjects who were diagnosed with sporadic hemiplegic migraine (SHM) and compared them with those of subjects with familial hemiplegic migraine (FHM) ${ }^{5}$ The symptoms of MUMS differ from those of genetically proved FHM and SHM. Unlike MUMS, which begins in the 30s, the onset of FHM and SHM is usually in the teens or occasionally the early 20s. Hemiplegic episodes of FHM and SHM have a more consistent occipital-frontal march of symptoms than MUMS, with visual (with typical fortification spectrum), sensory, language and motor symptoms. The duration of motor symptoms is typically shorter in FHM and SHM. $^{5}$ Despite these differences, if give-way weakness is interpreted as "true" weakness, many patients with MUMS meet the IHS definition of SHM.

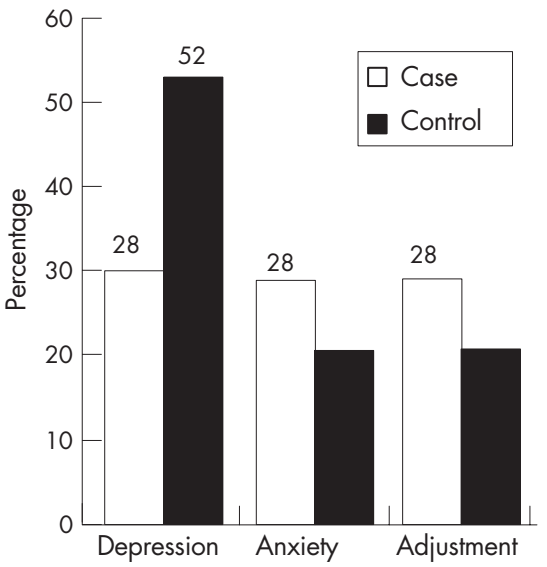

Figure 2 On psychiatric evaluation, depression (major depression, dysthymia or depressive disorder NOS, and adjustment disorder with depressed mood) was less common in patients with MUMS than in controls, although adjustment disorder with depressed mood was more common in cases than in controls $(p=N S)$. Adjustment disorder in this figure does not include adjustment disorder with depressed mood, which was allocated to the depression group.

Give-way weakness is considered by some to be evidence of a psychogenic disorder. ${ }^{6}$ This assumption may not be accurate. When examining the strength of a painful, injured limb, neurologists incorporate the give-way weakness into their interpretation of whether the patient's true strength is normal. ${ }^{6}$ Give-way weakness is similar to the clasp-knife weakness of spastic upper motor neurone injury. To explore the potential of psychological basis for the weakness, we compared the psychological state of cases and controls. Patients with MUMS did not differ from controls in any psychological measure. As expected, both cases and controls had a high burden of depression, both by interview and by BDI-II. ${ }^{7}$ Only conversion $\mathrm{V}$ showed a possible numerical difference between cases and controls, suggesting that patients with MUMS may have acquired an ability to publicly present a brave front while internally experiencing distress, but this was not statistically significant when corrected for multiple comparisons.

On balance, we favour the hypothesis that MUMS weakness is due to a disordered protective reflex similar to that which causes give-way weakness in an injured limb and which is related to the severe allodynia that accompanies MUMS. Allodynia, like aura, often spreads. ${ }^{8}$ Weakness has been correlated with allodynia in complex regional pain syndrome. ${ }^{9}$

Table 5 Beck Depression Inventory-II and migraine with unilateral motor symptoms scores

\begin{tabular}{llll}
\hline & $\begin{array}{l}\text { Cases } \\
\mathbf{n = 1 6 ( 6 6 . 7 \% )}\end{array}$ & $\begin{array}{l}\text { Controls } \\
\mathbf{n = 4 0}(83 \%)\end{array}$ & $\mathbf{p ~ V a l u { } ^ { * }}$ \\
\hline BDI score & $12.1(9.0)$ & $11.5(8.9)$ & 1.000 \\
Q9 (suicide question) & $13 / 3$ & $32 / 8$ & 1.000 \\
MMPI & $12(50 \%)$ & $29(60 \%)$ & \\
L & $55.4(8.5)$ & $52.8(8.8)$ & 1.000 \\
F & $47.6(0.9)$ & $51.5(10.2)$ & 1.000 \\
K & $49.9(10.8)$ & $52.9(9.2)$ & 1.000 \\
Gough & $5.3(13.6)$ & $-0.72(9.2)$ & 1.000 \\
M1 & $74.8(12.0)$ & $68.4(11.0)$ & 0.574 \\
M2 & $61.7(13.4)$ & $65.1(12.4)$ & 1.000 \\
M3 & $68.5(17.6)$ & $68.8(10.8)$ & 1.000 \\
M7 & $55.8(16.2)$ & $57.5(11.8)$ & 1.000 \\
Conversion V (Y/N) & $9 / 3$ & $11 / 18$ & 0.473 \\
\hline
\end{tabular}

BDI, Beck Depression Inventory.

*With Bonferroni correction for multiple comparisons. 
Fibromyalgia is often accompanied by weakness in a hemiparetic distribution. ${ }^{10}$

Our study has several weaknesses. The subjects of a retrospective study might be biased toward over-reporting symptoms. The validity and reliability of the concept of give-way weakness have not been carefully studied. Until an objective method to distinguish between true and give-way weakness is developed, this problem may be unavoidable. Our study occurred in a time of uncertainty about the most appropriate classification scheme for daily headache in patients with migraine. We used the 1996 Silberstein-Lipton criteria for transformed migraine, which now overlaps closely with the 2006 revised definition of chronic migraine. Despite these limitations, this study describes a group of patients with migraine with characteristic and consequential neurological symptoms that adversely affect their lives, add diagnostic uncertainties, cause significant expense and have no adequately determined aetiology.

\section{Authors' affiliations}

William B Young, Ronald S Kaiser, Thomas Jefferson University,

Philadelphia, Pennsylvania, USA

Kaanchan S Gangal, Jefferson Medical College, Thomas Jefferson

University, Philadelphia, Pennsylvania, USA
Raoul J Aponte, Philadelphia College of Osteopathic Medicine,

Philadelphia, Pennsylvania, USA

Competing interests: None declared.

\section{REFERENCES}

1 Thomsen LL, Eriksen MK, Romer SE, et al. An epidemiological survey of hemiplegic migraine. Cephalalgia 2002;22:361-75.

2 Couch JR, Stewart K, Leviston C, et al. Neurological symptoms associated with migraine are more common in women [abstract]. Headache 2003;45:546.

3 Shukla P, Richardson E, Young WB. Brush allodynia in an inpatient headache unit [abstract]. Headache 2003;43:542.

4 Fisher CM. Late life migraine accompaniments as a cause of unexplained transient ischemic attacks. Can J Neurol Sci 1980;7:9-17.

5 Thomsen LL, Olesen J. Sporadic hemiplegic migraine. Cephalalgia 2004;24:1016-23.

6 Guiloff RJ, Fruns M. Limb pain in migraine and cluster headache. J Neurol Neurosurg Psychiatry 2006;51:1022-31.

7 Verdugo RJ, Bell LA, Campero M, et al. Spectrum of cutaneous hyperalgesias/ allodynias in neuropathic pain patients. Acta Neurol Scand 2004; 1 10:368-76.

8 Rommel O, Gehling M, Dertwinkel R, et al. Hemisensory impairment in patients with complex regional pain syndrome. Pain 1999;80:95-101.

9 Headache Classification Committee. The international classification of headache disorders. 2nd edn. Cephalalgia 2004;24:1-160.

10 Lauritzen M. Pathophysiology of the migraine aura: the spreading depression theory. Brain 1994; 17:199-210.

11 Silberstein SD, Lipton RB, Dalessio DJ, eds. Wolff's headache and other head pain. 7th edn. New York: Oxford University Press, 2001.

\section{Take advantage of BMJ Journals' remarkable catalogue of titles with Related}

\section{Collections}

No busy professional has time to browse through all pertinent journals to find relevant articles, but with Related Collections you no longer have to. Follow the "Related Collections" link from any article and use the "Show Collections from other Journals" to expand your search across all BMJ Journals. Or simply follow the "Browse by topic" link on the home page. By setting up your own collections and receiving email alerts every time an article is added to your chosen area, you can build up your own significant body of knowledge. 